\title{
Colder freeze-in axinos decaying into photons
}

\author{
Kyu Jung Bae, ${ }^{1, *}$ Ayuki Kamada, ${ }^{1}$ Seng Pei Liew, ${ }^{2}$ and Keisuke Yanagi ${ }^{3}$ \\ ${ }^{1}$ Center for Theoretical Physics of the Universe, Institute for Basic Science (IBS), Daejeon 34126, Korea \\ ${ }^{2}$ Physik-Department, Technische Universität München, 85748 Garching, Germany \\ ${ }^{3}$ Department of Physics, University of Tokyo, Bunkyo-ku, Tokyo 113-0033, Japan
}

(Received 24 July 2017; revised manuscript received 2 November 2017; published 15 March 2018)

\begin{abstract}
We point out that $7 \mathrm{keV}$ axino dark matter (DM) in the R-parity violating (RPV) supersymmetric (SUSY) Dine-Fischler-Srednicki-Zhitnitsky axion model can simultaneously reproduce the $3.5 \mathrm{keV}$ x-ray line excess and evade stringent constraints from the Ly- $\alpha$ forest data. Peccei-Quinn symmetry breaking naturally generates both the TeV-scale $\mu$ term and the MeV-scale RPV term. The RPV term introduces a tiny axinoneutrino mixing and provides axino DM as a variant of the sterile neutrino DM explaining the $3.5 \mathrm{keV}$ x-ray line excess. Axinos are produced by freeze-in processes via the $\mu$ term. The resultant phase space distribution tends to be colder than the Fermi-Dirac distribution. The inherent entropy production from late-time saxion decay makes axinos even colder than those without saxion decay. The resultant axino DM takes the correct relic density and is compatible even with the latest and strongest constraint from the Ly- $\alpha$ forest data.
\end{abstract}

DOI: 10.1103/PhysRevD.97.055019

\section{INTRODUCTION}

An anomalous $3.5 \mathrm{keV}$ line excess in the XMM-Newton and Chandra x-ray spectra of the Andromeda galaxy and galaxy clusters was first reported by two independent groups [1]. Subsequent studies have shown that a similar excess is also found in the Galactic Center [2] and in the Suzaku data [3]. While there are reports of null detection, e.g., in dwarf spheroidal galaxies [4], the decaying dark matter (DM) explanation of the $3.5 \mathrm{keV}$ line excess is yet to be excluded (see Ref. [5] for a thorough review).

Sterile neutrino is a benchmark example of $7 \mathrm{keV}$ decaying DM [6]. In the simple model, its mass and mixing angle with active neutrino determine its phenomenology. On the other hand, there are two unsatisfactory points. First, the tiny mixing angle required for the $3.5 \mathrm{keV}$ line, $\sin ^{2} 2 \theta \sim 10^{-10}$, is yet to be theoretically motivated, and also it does not provide the correct relic abundance via the Dodelson-Widrow mechanism [7]. Second, even if other production mechanisms are introduced, the resultant sterile neutrino DM tends to be too hot to be compatible with the up-to-date Ly- $\alpha$ forest data [8]. In this article, we propose the R-parity violating (RPV) supersymmetric (SUSY) Dine-Fischler-Srednicki-Zhitnitsky (DFSZ) axion model as a solution to these problems. The fermion SUSY

\footnotetext{
*kyujungbae@ibs.re.kr
}

Published by the American Physical Society under the terms of the Creative Commons Attribution 4.0 International license. Further distribution of this work must maintain attribution to the author(s) and the published article's title, journal citation, and DOI. Funded by SCOAP. partner, axino $(\tilde{a})$, is a DM particle and can be regarded as sterile neutrino in phenomenology. ${ }^{1}$ The axino mass is of order of $\mathrm{keV}$ in some models [10], although it is naively of order of the gravitino mass since it is generated by the SUSY breaking.

In this model, the Peccei-Quinn (PQ) symmetry breaking at $v_{\mathrm{PQ}} \sim 10^{10} \mathrm{GeV}$ simultaneously generates the TeV-scale $\mu$ term via the Kim-Nilles mechanism [11] and MeV-scale bilinear R-parity violating (bRPV) term [12]. The $\mu$ term induces freeze-in production [13] of axino DM. The scalar SUSY partner of axion, saxion $(s)$, decays via the same interactions, leading to the late-time entropy production. The resultant axinos take the correct relic abundance. The bRPV term generates the tiny axino-neutrino mixing required for the $3.5 \mathrm{keV}$ line. Furthermore, TeV-scale SUSY resolves the hierarchy between the electroweak scale and the grand unification scale [14], although the hierarchy between the electroweak scale and the soft breaking scale has been highlighted by the null detection of SUSY particles at the Large Hadron Collider (LHC). TeV-scale SUSY also improves the grand unification of the standard model (SM) gauge couplings. Meanwhile, Peccei-Quinn (PQ) symmetry explains why quantum chromodynamics preserves $C P$ symmetry very accurately [15].

All the $7 \mathrm{keV}$ DM models proposed to explain the $3.5 \mathrm{keV}$ excess need to be revisited to examine whether their structure formation is compatible with the up-to-date

\footnotetext{
${ }^{1}$ Different realizations of $7 \mathrm{keV}$ axino DM decay were considered in Ref. [9]. Nevertheless, none of them discussed a phase space distribution of axino DM or Ly- $\alpha$ forest constraints.
} 
constraint from the Lyman- $\alpha$ forest data, $m_{\text {WDM }}>5.3 \mathrm{keV}$ [16]. Weaker constraints are also worth mentioning since the uncertainty in the reported constraint from the baryon complexity like the temperature evolution of intergalactic medium is in debate: $m_{\mathrm{WDM}}>2.0$ [17], 3.3 [18], and $4.09 \mathrm{keV}$ [19]. The constraints are reported in the conventional warm dark matter (WDM) model, but they can be converted to those on the other $7 \mathrm{keV}$ DM models once the phase space distribution function of DM particles is given, as demonstrated in the followings. The conventional WDM model assumes that WDM particles follow the Fermi-Dirac distribution with two spin degrees of freedom, and they reproduce the observed DM mass density by tuning the present temperature $\left(T_{\mathrm{WDM}, 0}\right)$ for a given mass $\left(m_{\mathrm{WDM}}\right)$,

$$
\Omega_{\mathrm{WDM}} h^{2}=\left(\frac{m_{\mathrm{WDM}}}{94 \mathrm{eV}}\right)\left(\frac{T_{\mathrm{WDM}, 0}}{T_{\nu, 0}}\right)^{3},
$$

where $T_{\nu, 0}$ is the present neutrino temperature. It follows that $T_{\mathrm{WDM}, 0}=0.1 T_{\nu, 0}$ for $m_{\mathrm{WDM}}=5.3 \mathrm{keV}$. Meanwhile, the present temperature is given by $T_{0}=$ $(10.75 / 106.75)^{1 / 3} T_{\nu, 0} \simeq 0.5 T_{\nu, 0}$ for $7 \mathrm{keV} \mathrm{DM}$ decoupled when the effective massless degrees of freedom count all the SM particles, $g_{*}=g_{\mathrm{SM}}=106.75$. The comparison of their naive warmness, $T_{0} / m$, reads as $\left(0.1 T_{\nu, 0} / 5.3 \mathrm{keV}\right)<$ $\left(0.5 T_{\nu, 0} / 7 \mathrm{keV}\right) .7 \mathrm{keV} \mathrm{DM}$ is hotter than the conventional WDM disfavored by the latest Ly- $\alpha$ forest data and results in a too smooth matter distribution of the Universe.

For a more robust analysis, we need to compare the linear matter power spectra by taking account of the phase space distribution of DM particles. We obtain the phase space distribution by integrating the Boltzmann equation and find that it is typically colder than the Fermi-Dirac one. The saxion also makes axino DM colder, since its late-time decay at $T_{D}^{s} \lesssim \mathcal{O}(100) \mathrm{GeV}$ injects a certain amount of entropy to the thermal bath after axino decoupling. We show that freeze-in $7 \mathrm{keV}$ axino DM is concordant with the current constraints from the Ly- $\alpha$ forest data.

\section{MODEL}

The DFSZ solution to the strong $C P$ problem invokes a coupling between a PQ symmetry breaking field $(X)$ and the up- and down-type Higgs doublets $\left(H_{u, d}\right)$ [20]. Its SUSY realization is given by the following superpotential,

$$
W_{\mathrm{DFSZ}}=\frac{y_{0}}{M_{*}} X^{2} H_{u} H_{d},
$$

where $y_{0}$ is a dimensionless constant and $M_{*}$ is a cutoff scale. The PQ charges of $X, H_{u}$, and $H_{d}$ are, respectively, $-1,1$, and 1 . Once the field $X$ develops its vacuum expectation value $(\mathrm{VEV}), X=\left(v_{\mathrm{PQ}} / \sqrt{2}\right) \exp \left(A / v_{\mathrm{PQ}}\right)$, where $A=(s+i a) / \sqrt{2}+\sqrt{2} \theta \tilde{a}+\theta^{2} \mathcal{F}_{A}$ is the axion superfield, the $\mu$ term and an axino interaction are generated as

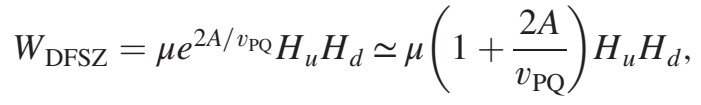

where $\mu=y_{0} v_{\mathrm{PQ}}^{2} /\left(2 M_{*}\right)$. The approximate equality is valid when considering the axino interaction. If $M_{*} \sim 10^{16} \mathrm{GeV}$, $y_{0} \sim 0.1$, and $v_{\mathrm{PQ}} \sim 10^{10} \mathrm{GeV}$, one finds $\mu \sim 500 \mathrm{GeV}$. This is a well-known solution to $\mu$-term generation by the Kim-Nilles mechanism [11]. From this interaction, freeze-in production of axinos occurs dominantly when the cosmic temperature $(T)$ is of order of the mass of the other SUSY particle involved in the process [21-23]. The contributions from dimension-five anomaly operators (e.g., axino-gluino-gluon) are suppressed [22].

The bRPV term is also generated as [12]

$$
W_{\mathrm{bRPV}}=\frac{y_{i}^{\prime}}{M_{*}^{2}} X^{3} L_{i} H_{u} \simeq \mu_{i}^{\prime}\left(1+\frac{3 A}{v_{\mathrm{PQ}}}\right) L_{i} H_{u} .
$$

If $M_{*} \sim 10^{16} \mathrm{GeV}, y_{i}^{\prime} \sim 1$, and $v_{\mathrm{PQ}} \sim 10^{10} \mathrm{GeV}$, one finds $\mu_{i}^{\prime} \sim \mathrm{MeV}$. This term generates an axino mixing with active neutrino. The mixing angle is given by

$$
|\theta| \simeq \frac{\mu^{\prime} v_{u}}{m_{\tilde{a}} v_{\mathrm{PQ}}} \simeq 10^{-5}\left(\frac{\mu^{\prime}}{4 \mathrm{MeV}}\right)\left(\frac{7 \mathrm{keV}}{m_{\tilde{a}}}\right)\left(\frac{10^{10} \mathrm{GeV}}{v_{\mathrm{PQ}}}\right),
$$

where $v_{u}$ is the VEV of $H_{u}$ and $m_{\tilde{a}}$ is the axino mass.

\section{FREEZE-IN PRODUCTION}

The axino production is governed by the following Boltzmann equation:

$\frac{d f_{\tilde{a}}(t, p)}{d t}=\frac{\partial f_{\tilde{a}}(t, p)}{\partial t}-\frac{1}{R(t)} \frac{d R(t)}{d t} p \frac{\partial f_{\tilde{a}}(t, p)}{\partial p}=\frac{1}{E} C(t, p)$,

where $f_{\tilde{a}}(t, p)$ is the axino phase space distribution as a function of the cosmic time $(t)$ and the axino momentum $(p), R(t)$ is the cosmic scale factor, and $C(t, p) / E$ is the collision term. Due to feeble interactions of the axino, one can safely neglect $f_{\tilde{a}}$ in the collision term. Then by integrating both sides from $t_{i}$ to $t_{f}$, one finds

$$
f_{\tilde{a}}\left(t_{f}, p\right)=\int_{t_{i}}^{t_{f}} d t \frac{1}{E} C\left(t, \frac{R\left(t_{f}\right)}{R(t)} p\right) .
$$

Once one collects all the relevant contributions to the collision term, it is easy to obtain the axino phase space distribution. We do not provide details here, but refer readers to Ref. [24].

For freeze-in production of axinos, the contributions of two-body and three-body decay, and $s$ - and $t$-channel scattering are taken into account. In Fig. 1, the phase space distributions, in form of $q^{2} f_{\tilde{a}}(q)\left(q=p_{\tilde{a}} / T_{\tilde{a}}\right)$, are 


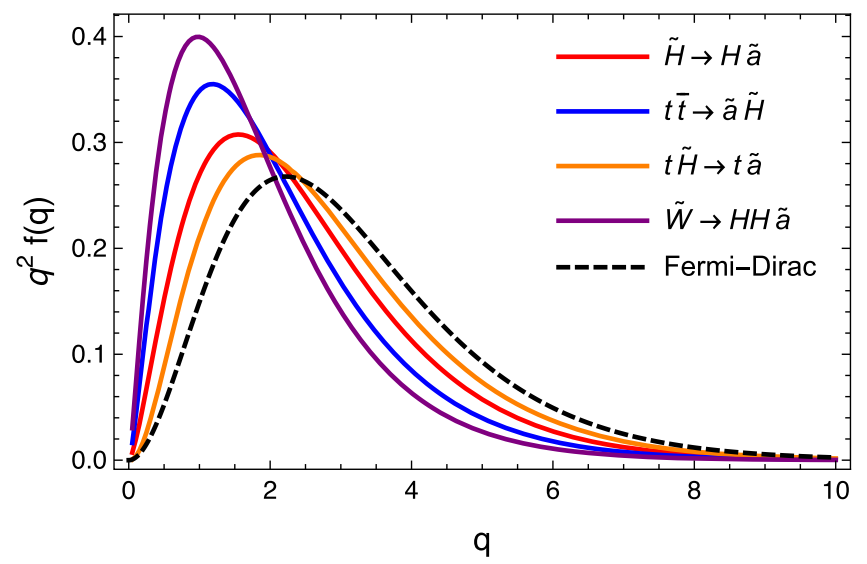

FIG. 1. Axino phase space distributions from respective production processes. The red, blue, and yellow solid lines show $q^{2} f(q)$ respectively from Higgsino two-body decay and $s$ - and $t$-channel scattering, while the purple solid line shows that from wino three-body decay. For comparison, the Fermi-Dirac distribution is shown by the dashed line. Each distribution is normalized such that $\int d q q^{2} f(q)=1$.

shown for Higgsino two-body decay $(\tilde{H} \rightarrow H+\tilde{a})$, $s$-channel scattering $(t+\bar{t} \rightarrow \tilde{H}+\tilde{a}), t$-channel scattering $(\tilde{H}+t \rightarrow \tilde{a}+t)$, and wino three-body decay $(\tilde{W} \rightarrow$ $H+H+\tilde{a})^{2}{ }^{2}$ Here we define the axino temperature by $T_{\tilde{a}}=\left(g_{*}(T) / g_{*}\left(T_{\text {th }}\right)\right)^{1 / 3} T$, where $T_{\text {th }}$ is set to the mass of the other SUSY particle involved in the freeze-in process. While all the freeze-in processes shown in Fig. 1 have a colder phase space distribution than the Fermi-Dirac distribution, the three-body decay case has the coldest phase space distribution. The reason is that three-body decay leads to a smaller kinetic energy of the final-state axino than the other processes at a given temperature. However, when one considers a realistic example, threebody decay rarely dominates the axino DM abundance so that the resulting axino phase space distribution follows those of two-body decay or $s$ - and $t$-channel scattering.

For a realistic analysis, we consider a benchmark scenario where the Higgsino-like neutralino is the nextto-lightest SUSY particle (NLSP). The mass spectrum is shown in Table I. This spectrum is preferable when one considers the TeV-scale SUSY with the Kim-Nilles solution to $\mu$ term and recent LHC constraints. ${ }^{3}$ In this

\footnotetext{
${ }^{2}$ In Fig. 1, we take top and Higgs to be massless, while introducing the thermal mass of intermediate Higgs in $t$-channel scattering. In the realistic analysis with the benchmark scenario below, we take into account the Higgs soft masses, but not the top mass since the freeze-in occurs before the electroweak phase transition.

${ }^{3} \mathrm{~A}$ relevant LHC experiment is the displaced Higgsino decay search with large missing energy. In our case, the relevant channel is displaced dijet plus missing energy (e.g., Ref. [25]) and the recasted lower bound on the Higgsino mass is around $400 \mathrm{GeV}$ [26].
}

TABLE I. MSSM parameters of the benchmark scenario with Higgsino NLSP is shown. The SM-like Higgs mass and soft masses at $Q=m_{\tilde{t}^{c}}$ are calculated by SUSY-HIT V1.5a [28]. The masses of all the other SUSY particles are taken to be $10 \mathrm{TeV}$.

\begin{tabular}{lcc}
\hline \hline Higgs VEV ratio & $\tan \beta$ & 20 \\
\hline$\mu$ term & $\mu$ & $500 \mathrm{GeV}$ \\
Wino mass & $M_{2}$ & $10 \mathrm{TeV}$ \\
$C P$-odd Higgs mass & $m_{A}$ & $10 \mathrm{TeV}$ \\
Stop masses & $m_{\tilde{Q}_{3}}=m_{\tilde{t}^{c}}$ & $6.5 \mathrm{TeV}$ \\
SM-like Higgs mass & $m_{h}^{\mathrm{SM}-\mathrm{like}}$ & $125 \mathrm{GeV}$ \\
$H_{u}$ soft mass & $m_{H_{u}}^{2}\left(Q=m_{\tilde{t}^{c}}\right)$ & $(956 \mathrm{GeV})^{2}$ \\
$H_{d}$ soft mass & $m_{H_{d}}^{2}\left(Q=m_{\tilde{t}^{c}}\right)$ & $(9.94 \mathrm{TeV})^{2}$ \\
\hline \hline
\end{tabular}

benchmark scenario, the dominant process is Higgs decay into Higgsino and axino, while Higgsino three-body decay and $s$ - and $t$-channel scattering also contribute to the relic density. Figure 2 shows the resultant axino phase space distribution accompanied by the contributions of the respective processes. ${ }^{4}$ It is clearly shown that freeze-in production of axinos leads to a colder phase space distribution than the Fermi-Dirac one. Note that in Fig. 2, the phase space distribution from two-body decay is colder than that in Fig. 1, since both Higgsino and Higgs are massive in the benchmark scenario. The phase space distribution from decay is colder for a more compressed mass spectrum [27], while that from scattering is not so sensitive to the mass spectrum and thus quickly dominates the relic density when the mass spectrum is more compressed. In the present axion model, on the other hand, the compressed mass spectum alone cannot make axinos cold enough to evade the up-todate constraint from the Ly- $\alpha$ date [24].

\section{Ly- $\alpha$ FOREST CONSTRAINTS}

In order to examine whether $7 \mathrm{keV}$ freeze-in axino DM with the phase space distribution obtained above is concordant with the constraints from the Ly- $\alpha$ forest data, we calculate linear matter power spectra by using a Boltzmann solver, CLASS [29]. We define the squared transfer function by the ratio of the WDM linear matter power spectrum to the cold dark matter one, which is denoted by $\mathcal{T}^{2}(k)$ as a function of the wave number, $k$. We regard a model as disfavored if $\mathcal{T}^{2}(k)$ falls below that for the lower bound of $m_{\text {WDM }}$ [8]. This is because such a model provides less seeds of structure of the Universe, and thus we expect that it ends up with the matter distribution of the Universe smoother than observed in the Ly- $\alpha$ forest data even after the nonlinear growth. Figure 3 compares $\mathcal{T}^{2}(k)$ in the

\footnotetext{
${ }^{4}$ We add the $s$-channel scattering contribution to that of twobody decay. This is because we define the $s$-channel scattering contribution by subtracting the Higgs pole from the matrix element to avoid the double counting of two-body decay. See Ref. [24] for details.
} 


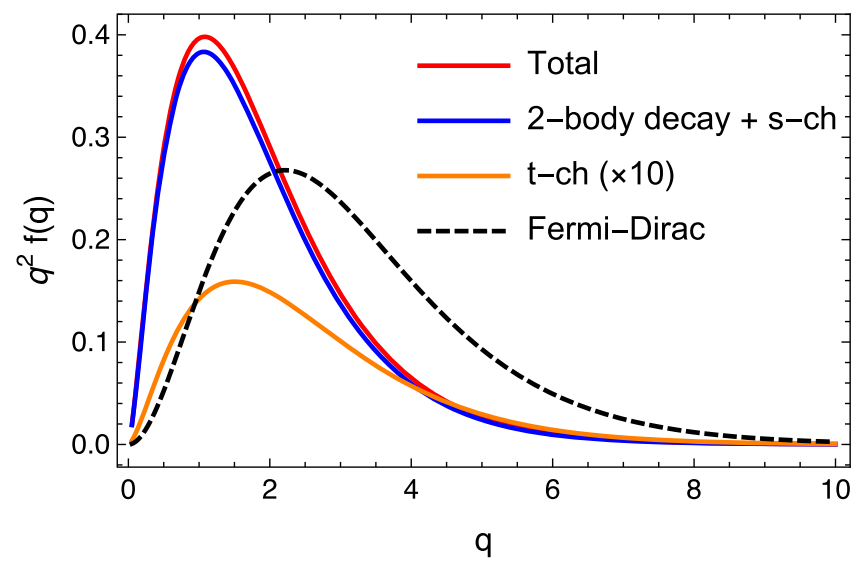

FIG. 2. Axino phase space distributions for the benchmark scenario. The red solid line shows the total axino phase space distribution normalized such that $\int d q q^{2} f(q)=1$. The blue solid line is sum of the contributions from Higgs two-body decay and Higgsino $s$-channel scattering, and the yellow solid line is the contribution from Higgsino $t$-channel scattering (multiplied by 10 for visualization). The normalized Fermi-Dirac distribution is shown by the dashed line.

benchmark scenario with those for the Ly- $\alpha$ forest lower bounds of $m_{\mathrm{WDM}}=2.0,3.3,4.09$, and $5.3 \mathrm{keV}$. For comparison, we also show $\mathcal{T}^{2}(k)$ for $7 \mathrm{keV}$ axino DM from UV production via nonrenormalizable operators (more specifically, $\tilde{W}+H \rightarrow H+\tilde{a}$ ), where the phase space distribution of axinos is similar to the Fermi-Dirac distribution. It is clearly shown that $7 \mathrm{keV}$ axino DM from UV production is disfavored by the Ly- $\alpha$ forest data even

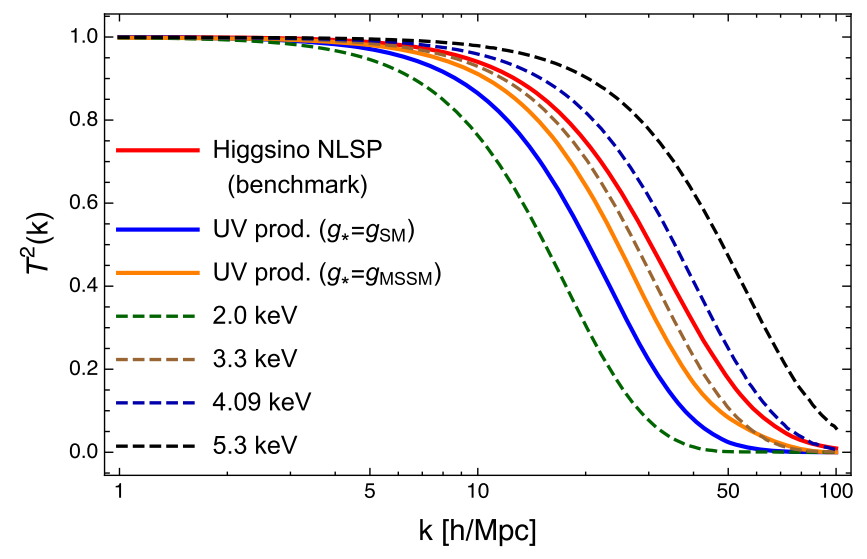

FIG. 3. Squared transfer functions for $7 \mathrm{keV}$ axino DM and Ly- $\alpha$ forest constraints. The red solid line shows $\mathcal{T}^{2}(k)$ in the benchmark scenario, and the blue (yellow) solid line shows $\mathcal{T}^{2}(k)$ for axino DM from UV production via nonrenormalizable operators with $g_{*}=g_{\mathrm{SM}}=106.75\left(g_{*}=g_{\mathrm{MSSM}}=226.75\right)$. The dashed lines show $\mathcal{T}^{2}(k)$ in the conventional WDM model with the Fermi-Dirac distribution and the temperature being fixed such that the relic density reproduces the observed DM mass density. Below the dashed lines, the models provide less structure than observed in the Ly- $\alpha$ forest data, and consequently models that fall below can be ruled out.

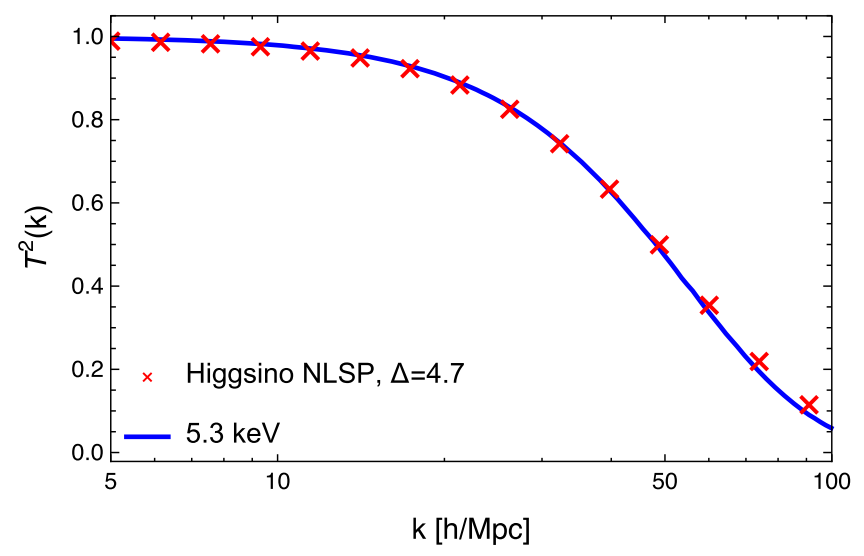

FIG. 4. Squared transfer functions with the entropy production from late-time saxion decay. Red crossed points show $\mathcal{T}^{2}(k)$ for the benchmark scenarios with $\Delta=4.7$. Blue solid line shows $\mathcal{T}^{2}(k)$ for $m_{\mathrm{WDM}}=5.3 \mathrm{keV}$ corresponding to the most stringent lower bound from the Ly- $\alpha$ forest data.

for $g_{*}=g_{\mathrm{MSSM}}=226.75$, when one adopts $m_{\mathrm{WDM}}>$ $3.3 \mathrm{keV}$ or the stronger constraints. $7 \mathrm{keV}$ axino DM from freeze-in production in our benchmark scenario is allowed by $m_{\mathrm{WDM}}>3.3 \mathrm{keV}$. It is, however, still in tension with the stronger constraint, $m_{\mathrm{WDM}}>4.09 \mathrm{keV}$.

In this regard, one can conclude that a certain amount of entropy production is still necessary, when the stronger Ly- $\alpha$ forest constraints, $m_{\mathrm{WDM}}>4.09$ and $5.3 \mathrm{keV}$, are taken into account. In Fig. 4, we find that $7 \mathrm{keV}$ axino DM with entropy dilution factor $\Delta=4.7$ fits the strongest lower bound from the Ly- $\alpha$ forest data, $m_{\mathrm{WDM}}=5.3 \mathrm{keV}$, very well. ${ }^{5}$ It means that we need only a mild entropy dilution factor, $\Delta>4.7$, to evade the Ly- $\alpha$ forest constraints.

In the SUSY DFSZ model, such an entropy dilution factor is naturally achieved by late-time saxion decay. Saxions are abundantly produced in the form of coherent oscillation. The yield is given by

$Y_{s}^{\mathrm{CO}} \simeq 1.9 \times 10^{-6}\left(\frac{\mathrm{GeV}}{m_{s}}\right)\left(\frac{\min \left[T_{R}, T_{s}\right]}{10^{7} \mathrm{GeV}}\right)\left(\frac{s_{0}}{10^{12} \mathrm{GeV}}\right)^{2}$,

where $m_{s}$ is the saxion mass, $T_{R}$ is the reheat temperature, $s_{0}$ is the saxion initial amplitude, and $T_{s}$ is determined by $\left.(3 / R)(d R / d t)\right|_{T=T_{s}}=m_{s}$. Such saxions dominate the energy density of the Universe at

$T_{e}^{s} \simeq 2.5 \times 10^{2} \mathrm{GeV}\left(\frac{\min \left[T_{R}, T_{s}\right]}{10^{7} \mathrm{GeV}}\right)\left(\frac{s_{0}}{10^{16} \mathrm{GeV}}\right)^{2}$.

If the saxion domination occurs at $T_{e}^{s} \simeq 250 \mathrm{GeV}$ and decay occurs at $T_{D}^{s} \lesssim 53 \mathrm{GeV}$, it is possible to obtain the

\footnotetext{
${ }^{5}$ We can infer the required entropy dilution factor by comparing the velocity dispersions [24,30].
} 
entropy dilution factor $\Delta \gtrsim 4.7$ which is determined by the temperature ratio as $\Delta=T_{e}^{s} / T_{D}^{s}$ [31]. $T_{D}^{s} \simeq 53 \mathrm{GeV}$ is realized when the saxion with $m_{s}=110 \mathrm{GeV}$ decays dominantly into $b \bar{b}$ via the $\mu$ term, and $v_{\mathrm{PQ}}=2.5 \times 10^{10} \mathrm{GeV}$ [32]. Consequently, we find that the total mass density of $7 \mathrm{keV}$ axinos also meets the observed DM one, i.e.,

$$
\Omega_{\tilde{a}} h^{2} \simeq 0.1\left(\frac{4.7}{\Delta}\right)\left(\frac{2.5 \times 10^{10} \mathrm{GeV}}{v_{\mathrm{PQ}}}\right)^{2}\left(\frac{m_{\tilde{a}}}{7 \mathrm{keV}}\right) .
$$

\section{CONCLUSION}

We have proposed $7 \mathrm{keV}$ axino DM in the RPV SUSY DFSZ axion model as a variant of the sterile neutrino DM explaining the $3.5 \mathrm{keV} x$-ray line excess. The model has two advantages over the simple sterile neutrino model. First, it gives a natural explanation to the tiny axino-active neutrino mixing preferred by the $3.5 \mathrm{keV}$ $\mathrm{x}$-ray line excess, and also provides freeze-in production of axino DM. Second, it is concordant with the severest constraint from the Ly- $\alpha$ forest data with the help of the colder phase space distribution resulting from freezein production and the larger SM-to-DM temperature ratio due to mild entropy production from late-time saxion decay that is naturally accessible in the model. We stress that, even with entropy production, the whole DM density is explained and dominated by the freeze-in axinos.

We have emphasized that our ultraviolet model naturally accommodates $\mathrm{keV}$-scale decaying dark matter in terms of axino. It, in turn, follows that a combination of x-ray observations and $\mathrm{Ly}-\alpha$ forest data infers fundamental quantities of the ultraviolet model such as the ratio of the PQ symmetry breaking scale to the cutoff scale and the saxion mass. These hints constrain the underlying PQ symmetry breaking sector and its communication with the SUSY breaking sector.

\section{ACKNOWLEDGMENTS}

The work of K. J. B. and A. K. was supported by IBS under the Project Code IBS-R018-D1. S. P. L. has received support from the European Union's Horizon 2020 research and innovation programme under the Marie Curie grant agreement, Contract No. 675440. A. K. and S. P. L. would like to acknowledge the Mainz institute for Theoretical Physics (MITP) where this work was initiated.
[1] A. Boyarsky, O. Ruchayskiy, D. Iakubovskyi, and J. Franse, Phys. Rev. Lett. 113, 251301 (2014); E. Bulbul, M. Markevitch, A. Foster, R. K. Smith, M. Loewenstein, and S. W. Randall, Astrophys. J. 789, 13 (2014).

[2] A. Boyarsky, J. Franse, D. Iakubovskyi, and O. Ruchayskiy, Phys. Rev. Lett. 115, 161301 (2015).

[3] O. Urban, N. Werner, S. W. Allen, A. Simionescu, J. S. Kaastra, and L. E. Strigari, Mon. Not. R. Astron. Soc. 451, 2447 (2015).

[4] D. Malyshev, A. Neronov, and D. Eckert, Phys. Rev. D 90, 103506 (2014).

[5] D. Iakubovskyi, Adv. Astron. Space Phys. 6, 3 (2016).

[6] K. N. Abazajian et al., arXiv:1204.5379.

[7] S. Dodelson and L. M. Widrow, Phys. Rev. Lett. 72, 17 (1994); K. Abazajian, Phys. Rev. D 73, 063506 (2006); T. Asaka, M. Shaposhnikov, and M. Laine, J. High Energy Phys. 01 (2007) 091; J. High Energy Phys. 02, 028(E) (2015).

[8] A. Merle and A. Schneider, Phys. Lett. B 749, 283 (2015); A. Schneider, J. Cosmol. Astropart. Phys. 04 (2016) 059; J. König, A. Merle, and M. Totzauer, J. Cosmol. Astropart. Phys. 11 (2016) 038.

[9] J. C. Park, K. Kong, and S. C. Park, Phys. Lett. B 733, 217 (2014); K. Y. Choi and O. Seto, Phys. Lett. B 735, 92 (2014); S. P. Liew, J. Cosmol. Astropart. Phys. 05 (2014) 044.

[10] T. Goto and M. Yamaguchi, Phys. Lett. B 276, 103 (1992); E. J. Chun, J. E. Kim, and H. P. Nilles, Phys. Lett. B
287, 123 (1992); E. J. Chun and A. Lukas, Phys. Lett. B 357, 43 (1995).

[11] J.E. Kim and H.P. Nilles, Phys. Lett. 138B, 150 (1984).

[12] E. J. Chun, Phys. Lett. B 454, 304 (1999); K. Choi, E. J. Chun, and K. Hwang, Phys. Rev. D 64, 033006 (2001); E. J. Chun and H. B. Kim, J. High Energy Phys. 10 (2006) 082.

[13] L. J. Hall, K. Jedamzik, J. March-Russell, and S. M. West, J. High Energy Phys. 03 (2010) 080.

[14] H. P. Nilles, Phys. Rept. 110, 1 (1984); H. E. Haber and G. L. Kane, Phys. Rep. 117, 75 (1985); S. P. Martin, Adv. Ser. Dir. High Energy Phys. 21, 1 (2010); 18, 1 (1998).

[15] R. D. Peccei and H. R. Quinn, Phys. Rev. Lett. 38, 1440 (1977); R. D. Peccei and H. R. Quinn, Phys. Rev. D 16, 1791 (1977); S. Weinberg, Phys. Rev. Lett. 40, 223 (1978); F. Wilczek, Phys. Rev. Lett. 40, 279 (1978).

[16] V. Iršič et al., Phys. Rev. D 96, 023522 (2017).

[17] M. Viel, J. Lesgourgues, M. G. Haehnelt, S. Matarrese, and A. Riotto, Phys. Rev. D 71, 063534 (2005).

[18] M. Viel, G. D. Becker, J. S. Bolton, and M. G. Haehnelt, Phys. Rev. D 88, 043502 (2013).

[19] J. Baur, N. Palanque-Delabrouille, C. Yche, C. Magneville, and M. Viel, J. Cosmol. Astropart. Phys. 08 (2016) 012.

[20] M. Dine, W. Fischler, and M. Srednicki, Phys. Lett. 104B, 199 (1981); A. R. Zhitnitsky, Yad. Fiz. 31, 497 (1980) [Sov. J. Nucl. Phys. 31, 260 (1980)]. 
[21] E. J. Chun, Phys. Rev. D 84, 043509 (2011).

[22] K. J. Bae, K. Choi, and S. H. Im, J. High Energy Phys. 08 (2011) 065.

[23] K. J. Bae, E. J. Chun, and S. H. Im, J. Cosmol. Astropart. Phys. 03 (2012) 013.

[24] K. J. Bae, A. Kamada, S. P. Liew, and K. Yanagi, J. Cosmol. Astropart. Phys. 01 (2018) 054.

[25] M. Aaboud et al. (ATLAS Collaboration), arXiv:1710.04901.

[26] G. Barenboim, E. J. Chun, S. Jung, and W. I. Park, Phys. Rev. D 90, 035020 (2014).

[27] J. Heeck and D. Teresi, Phys. Rev. D 96, 035018 (2017).
[28] M. M. Muhlleitner, A. Djouadi, and M. Spira, Acta Phys. Pol. B 38, 635 (2007).

[29] D. Blas, J. Lesgourgues, and T. Tram, J. Cosmol. Astropart. Phys. 07 (2011) 034; J. Lesgourgues and T. Tram, J. Cosmol. Astropart. Phys. 09 (2011) 032.

[30] A. Kamada, N. Yoshida, K. Kohri, and T. Takahashi, J. Cosmol. Astropart. Phys. 03 (2013) 008.

[31] E. W. Kolb and M. S. Turner, Front. Phys. 69, 1 (1990).

[32] K. J. Bae, H. Baer, and E. J. Chun, J. Cosmol. Astropart. Phys. 12 (2013) 028. 